\section{Alkoholabhängigkeit}

Die EMA hat eine Empfehlung für die Zulassung von Nalmefen (Selincro ${ }^{\circledR}$ ) zur Reduktion des Alkoholkonsums bei Erwachsenen mit Alkoholabhängigkeit, deren Alkoholkonsum sich auf einem hohen Risikoniveau befindet, ausgesprochen. Die Empfehlung basiert auf den Ergebnissen dreier randomisierter, placebokontrollierter Doppelblindstudien, in denen die Wirkung von $18 \mathrm{mg}$ Nalmefen bei Erwachsenen mit Alkoholabhängigkeit gezeigt wurde. Im Falle der Zulassung erwartet der Hersteller die Markteinführung in Europa ab Mitte 2013.

Nach Informationen von Lundbeck

\section{Adulte ADHS}

Seit der Zulassung des retardierten Methylphenidat-Präparates Medikinet ${ }^{\oplus}$ adult können auch erwachsene Patienten mit ADHS von dieser Substanz profitieren. Die Number Needed to Treat (NNT) für Methylphenidat (MPH) bei Erwachsenen mit ADHS liegt bei 3 [Faraone SV et al. J Atten Disord 2010; 13: 532-38].,Ein ungewöhnlich positiver Wert", wie Professor Michael Rösler, Homburg/Saar auf einem MediceSymposium beim DGPPN-Kongress 2012 hervorhob. Für andere etablierte Psychopharmaka wie Neuroleptika oder Antidepressiva sei die NNT um das zwei- bis dreifache höher. „Um eine optimale Symptomkontrolle zu erzielen, ist es wichtig, MPH in ausreichender Dosis zu applizieren", erklärte Rösler. Nach den Daten der EMMAStudie führe zwar auch niedrig dosiertes MPH $(0,55 \mathrm{mg} / \mathrm{kg})$ zu einer signifikanten und stabilen Symptomreduktion [Rösler M et al. Eur Arch Psychiatry Clin Neurosci 2009; 259: 120-29]. In der Folgestudie QUMEA sei es unter höheren Dosierungen $(1 \mathrm{mg} / \mathrm{kg}$ ) jedoch zu einer noch ausgeprägteren Verbesserung der ADHS-Symptomatik und einem zusätzlichen günstigen Einfluss auf das funktionelle Therapieergebnis gekommen [Retz $\mathrm{W}$ et al. World J Biol Psychiatry 2012; 13: 48-59]. Außerdem sei im MPH-Arm der QUMEAStudie eine signifikante Verbesserung zahlreicher funktioneller sowie psychopathologischer Parameter beobachtet worden. Auch nach längerer Behandlungsdauer habe die Wirkung nicht nachgelassen.

Nach Informationen von Medice

\title{
Die innere Uhr resynchronisieren
}

- Die Depression ist eine chronobiologische Erkrankung, die häufig mit einer gestörten 24-Stunden-Rhythmik assoziiert ist, erklärte Professor Göran Hajak, leitender Neurologe und Psychiater am Bamberger Klinikum am Michelsberg. Der Schlaf-WachRhythmus sei bei depressiven Patienten häufig verflacht und phasenverschoben. Das Ausmaß der zirkadianen Dysregulation korreliere dabei mit dem Schweregrad der Depressivität.

Einen viel versprechenden neuen Ansatz in der Behandlung der Major Depression sieht Hajak deshalb in Agomelatin (Valdoxan ${ }^{\circledast}$ ). Der Melatoninagonist und 5- $\mathrm{HT}_{2 \mathrm{C}}$-Antagonist zeigte in klinischen Studien neben der antidepressiven Wirksamkeit auch deutliche Verbesserungen der Schlafqualität bei Erhalt der Aktivität am Tage, ohne die Schlafarchitektur zu verändern. Dass sich Agomelatin auch in der täglichen Praxis bewährt, belegte eine große deutsche nicht-interventionelle Studie mit mehr als 3.300 Patienten. Erste Daten einer Subgruppenanalyse, die Professor Gerd Laux, Psychiater aus Wasserburg, vorstellte, zeigen auch eine rasche und anhaltende Wirkung bei schwerer Depression und Altersdepression. Bereits in den ersten zwei Wochen besserten sich die Symptome auf der Montgomery-Asberg Depression Rating Scale (svMADRS) bei etwa jedem zweiten Teilnehmer um mindestens $20 \%$. Nach dreimonatiger Behandlung erreichten $48 \%$ beziehungsweise $59 \%$ der beiden Gruppen eine Remission. Die meisten Ärzte bewerteten die Verträglichkeit von Agomelatin als sehr gut oder gut, Nebenwirkungen (bei ca. $10 \%)$ waren meist milde und unspezifisch. Wegen der Möglichkeit eines Transaminasenanstiegs sind Leberenzymkontrollen bei Therapiebeginn, nach drei, sechs, zwölf und 24 Wochen durchzuführen. Als positiv wertete Laux die Gewichtsneutralität der Substanz und den Erhalt der sexuellen Funktion.

Dr. Martina-Jasmin Utzt

Satellitensymposium „Depression: Eine komplexe systemische Erkrankung" beim DGPPN-Kongress, Berlin, 22.11.2012 Veranstalter: Servier

\section{Gleichmäßige Wirkspiegel über 24 Stunden erreichen}

— Für eine gute Symptomkontrolle sind bei der Parkinson-Krankheit möglichst gleichmäßige Wirkstoffspiegel essenziell. Mit retardiertem Ropinirol in einer speziellen Galenik lassen sich sowohl motorische als auch nichtmotorische Symptome über 24 Stunden hinweg gut kontrollieren.

Ein Problem gerade bei Patienten mit fortgeschrittener Parkinson-Erkrankung sind schwankende Serumspiegel der dopaminergen Arzneien. Haben die Wirkstoffspiegel ihr Maximum erreicht, treten oft Nebenwirkungen wie Dyskinesien auf; sinken sie vor der nächsten Einnahme unter einen bestimmten Wert, wird die Motorik nicht mehr ausreichend kontrolliert. Dies kann gerade auch nachts zum Problem werden, erinnerte Professor Heinz Reichmann, Dresden. So wurden in Studien Schlafstörungen unter den vier Symptomen genannt, welche die Patienten am meisten belasten. Auch eine morgendliche Akinese durch niedrige dopaminerge Wirkspiegel könne die Patienten deutlich beeinträchtigen.
Um Wirkungsfluktuationen zu vermeiden und Off-Phasen nicht nur tagsüber, sondern auch nachts oder frühmorgens zu minimieren, sei eine möglichst kontinuierliche Rezeptorstimulation durch retardierte Präparate von Vorteil. Maßstäbe setze hier der Dopaminagonist Ropinirol in der Retardformulierung (ReQuip-Modutab ${ }^{\circledast}$ ). Reichmann zeigte Daten, nach denen die einmal tägliche Einnahme von $8 \mathrm{mg}$ des retardierten Wirkstoffs zu deutlich niedrigeren Plasmaspitzen und wesentlich gleichmäßigeren Serumkonzentrationen führt als die dreimal tägliche Einnahme von jeweils 2,5 mg nicht-retardiertem Ropinirol. Nach 24 Stunden sind mit dem Retardpräparat die Serumwerte noch etwa doppelt so hoch wie mit nicht-retardiertem Wirkstoff - und damit auch nachts oft noch ausreichend. Dieser Vorteil mache sich auch in der Schlafqualität bemerkbar, so Reichman. Auf der Parkinson's Disease Sleep Scale (PDSS) verbesserte sich der Wert in Studien von knapp 100 Punkten mit dem retardierten Ropinirol im Vergleich zu Place- 